Part III

\title{
Observations and Results
}




\title{
Photographic Observation of Stellar Occultations
}

\author{
A. Tomić ${ }^{1}, N$. Čabrić ${ }^{1}$ and $V . \check{C C}$ elebonovic $^{2}$ \\ 1 Narodna Opservatorija, Kalemegdan, 11000 Beograd, Yugoslavia \\ 2 Institute of Physics, POB 57,11001 Beograd, Yugoslavia
}

Stellar occultations are usually observed visually, but this can also be done photographically. Our method has some advantages :

- the obtained moments of contacts refer to the smoothed lunar limb,

- the error in the determination of contacts is calculable,

- the error may be less than in visual work,

- the reduction of photographs is fast, due to the application of an "on-line" computer [1].

In order to achieve all this, it is necessary to make a series of photographs of the Moon and the object being occulted before the first and after the last contact. This is a crucial step in our method, because the photographs must be of high quality. This demands two things :

- the images of the occulted object and the Moon must be reliably recorded and

- the illuminated limb of the Moon must give optimal darkening on the film.

The required exposure time is given by [2] :

$$
t_{M}=\frac{(F / D)^{2}}{\tau_{0} S} \frac{1}{1600 \tau_{a} A b(\lambda, \psi)}
$$

and for the star :

$$
t_{S}=\frac{(F / D)^{2}}{\tau_{0} S}\left(C_{o} C_{S} / L F\right)^{2} 10^{(0.4 m+0.67)}
$$

where both time intervals are in seconds, $D$ is the diameter of the objective whose focal length is $F, \tau_{o}$ and $\tau_{a}$ are the transmitances of the optical equipment and the atmosphere $(\leq 1), S$ is the sensitivity of the photographic emulsion (ASA), A is the albedo, $m$ the photographic stellar magnitude, $b(\lambda, \psi)$ is the distribution function of the brigthness on the Moon ( $\lambda$ the selenographic longitude and $\psi$ the lunar phase angle), $\mathrm{L}(\mathrm{N})$ is the resolving power of the objective (film) $\left(\mathrm{mm}^{-1}\right), \mathrm{C}_{S}=1+\mathrm{L} / \mathrm{N}$ characterizes the reduction of the resolving power, and $\mathrm{C}_{o}$ is a numerical parameter, describing the atmospheric spreading ( $\left.\Delta^{\prime \prime}\right)$ of the point image : $\mathrm{C}_{o}=\Delta /(1+\mathrm{kD} / \mathrm{FN})$. For a panchromatic film, $\mathrm{k}=1500 \mathrm{~mm}^{-1}$. 
Therefore, the exposure time is adjusted to the Moon, and the continuously variable parameter will be the age of the Moon. It is important to determine the limiting stellar magnitude attainable. It follows from eqs. (1) and (2) that :

$$
m=1.875-2.5 \log (A b(\lambda, \psi))-2.5 \log \left(r_{a} C_{o}^{2} \delta^{2}(1+k D / F N)^{2}\right)
$$

where $\delta$ is radius of the Airy disk.

The limiting magnitude increases with increase in $\mathrm{D} / \mathrm{F}, \mathrm{S}, \tau_{a}, C_{o}$. At the same time the exposure time becomes shorter. A greater value of $\mathrm{N}$ gives a larger $m$, but it demands longer exposure. Worse observing conditions (larger $\mathrm{C}_{0}$ ) increase the exposure time and reduce $m$.

An example, calculated for a Zeiss 110/2000-mm refractor, with $S=32, \tau_{0}=0.8, \tau_{a}=0.5$, $\mathrm{C}_{o}=1$ is shown in the figure. Note that the point $\mathrm{d}=14$ corresponds to New Moon.

Table I - Some values of the function $\mathrm{b}(90, \phi)$

$\begin{array}{lllllllll}\text { age (days) } & 1 & 4 & 8 & 12 & 16 & 20 & 24 & 28 \\ \mathrm{~b}(90, \psi) & 0.02 & 0.34 & 0.69 & 0.94 & 0.97 & 0.81 & 0.49 & 0.08\end{array}$

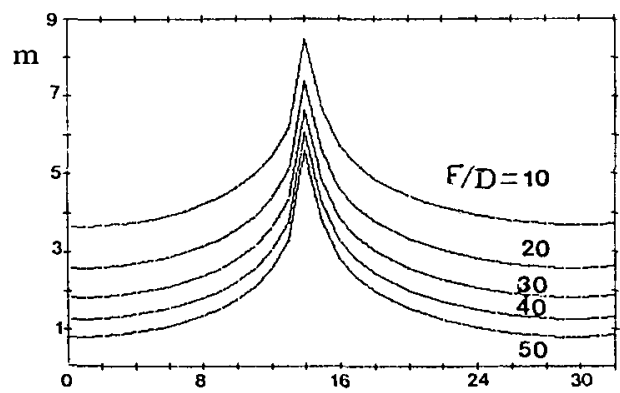

References :

1. Cabrić, N., Tomić, A. and Čelebonović, V., 1985, Publ. Obs. Astron. Belgrade, $\underline{33}, 75$.

2. Tomić, A., 1983, Astrophotography, Astron. Soc. of the University, Sarajevo, Yugoslavia (in Serbo-croat). 